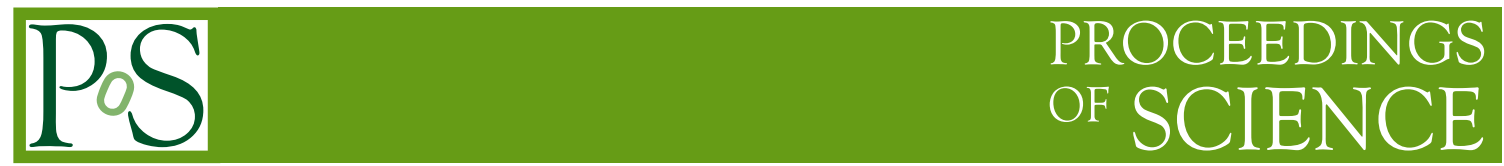

\title{
Quarkonium: Experimental Overview
}

\section{J. Matthew Durham ${ }^{a, *}$}

${ }^{a}$ Los Alamos National Laboratory,

Los Alamos, NM, USA

E-mail: durham@lanl.gov

The study of the bound states of heavy quarks in a QCD medium is an important foundation of heavy ion physics. Two major experiments at the Relativistic Heavy Ion Collider (PHENIX and STAR) and four major experiments at the Large Hadron Collider (ALICE, ATLAS, CMS, and $\mathrm{LHCb}$ ) are all producing new results and refining previous observations with unprecedented precision and kinematic reach. In addition, measurements of the interactions of exotic heavy quark states with a QCD medium are becoming accessible for the first time. The proceedings discuss new results and remaining puzzles in quarkonium measurements, the first experimental results on exotic hadrons in the QCD medium, and give a brief outlook on future facilities.

Hard Probes 2020

1-6 June 2020

Austin, Texas

${ }^{*}$ Speaker 


\section{Introduction}

Among the many successes of the quark model of hadron structure is the ability to predict the observed spectrum of heavy quarkonia, which are bound states of $c \bar{c}$ or $b \bar{b}$ quarks. The allowed bound states of two heavy quarks $Q$ and $\bar{Q}$ can be found by solving the Schrödinger equation with the QCD potential

$$
V_{Q \bar{Q}}(r)=-\frac{4}{3} \frac{\alpha_{s}}{r}+b r+\frac{32 \pi \alpha_{s}}{9 m_{Q}^{2}} \delta_{\sigma}(r) \overrightarrow{S_{Q}} \cdot \overrightarrow{S_{\bar{Q}}}
$$

where the first term is a color Coulomb interaction that dominates at short distances, the second term enforces QCD confinement at large distances, and the third term describes the spin-spin hyperfine interaction between the two quarks [1]. The predicted masses of measured quarkonia states are in good agreement with these calculations; for example, the most recently observed quarkonium state, the $\psi_{3}\left(1^{3} \mathrm{D}_{3}\right)$ found by LHCb in 2019 , has a mass within $1 \%$ of the potential model calculation [2]. The rich structure of $Q \bar{Q}$ states is accessible experimentally and well-understood theoretically.

In heavy ion collisions (and perhaps even high-multiplicity $p+p$ collisions) this rich structure is embedded inside an evolving QCD medium, where a variety of effects come into play. The dominant effect can vary in different density and temperature regimes, see Fig. 1 for a drawing. In a relatively diffuse medium, interactions between the $Q \bar{Q}$ pair and other nearby particles can disrupt formation of the state before or after hadronization. Heavy quarks can participate in hydrodynamic flow along with the light quarks in the system, which can be measured as an azimuthal anisotropy (attributed to initial state effects in some models). If the temperature of the medium is above the deconfinement temperature, the presence of free color charges can inhibit $Q \bar{Q}$ pair formation or dissociate formed pairs. Finally, at the extremes of density achieved in central AA collisions, a new quarkonium production mechanism becomes important, where deconfined heavy quarks can coalesce at freezeout into quarkonia states. Experimentally, the environments where different effects dominate can be prepared by varying collider energies, beam species, and kinematic regions where measurements are made.

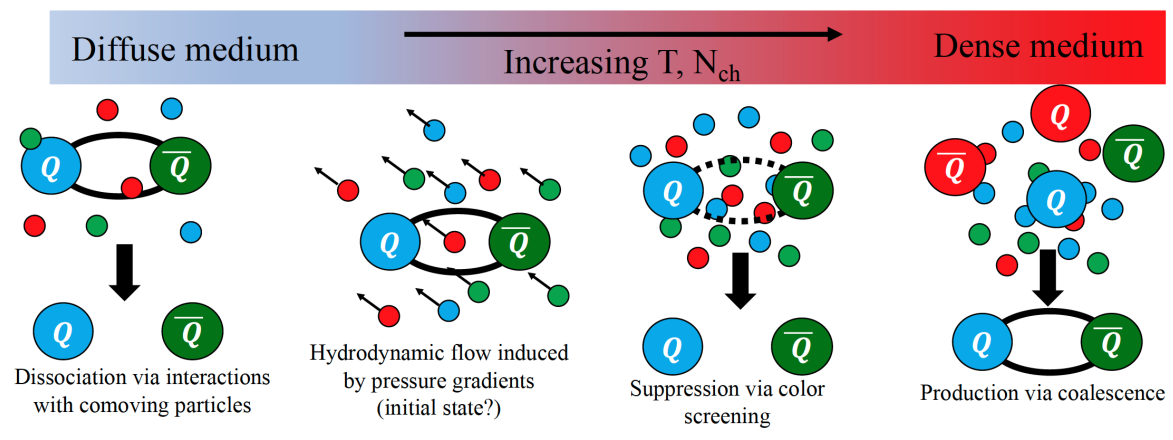

Figure 1: A range of effects come into play as the temperature and density of the QCD medium varies. 


\section{Quarkonium in Medium}

We begin with a discussion of recent results from the ALICE collaboration. A close look at $J / \psi$ production in $p+p$ collisions at $13 \mathrm{TeV}$ reveals a significant increase of the $J / \psi$ yield with charged particle multiplicity [3] as shown in Fig. 2. Various models based on, for example, coherent particle production [4] or color-glass condensate (CGC) effects [5, 6] are able to describe the data.

It is illustrative to compare multiplicity dependent effects on $J / \psi$ with the $\psi(2 S)$ state, which has the same $c \bar{c}$ quark content but a different binding energy. As such, the initial state effects described by the calculations which reproduce $J / \psi$ multiplicity dependence should give a nearly identical behavior for the $\psi(2 S)$. However, the ratio of $\psi(2 S) / J / \psi$ production may show some evidence for a decreasing trend with multiplicity, as shown in the right panel of Fig. 2. Any decrease must be therefore be due to final state effects which occur after the $c \bar{c}$ pair has formed a color neutral hadron. A calculation based on the Comover Interaction Model [7] shows a trend similar to the data, but may over-estimate the suppression at the highest multiplicity. Additional data with improved uncertainties is needed to make firm statements about final state effects on $\psi(2 S)$ in $p+p$ collisions.
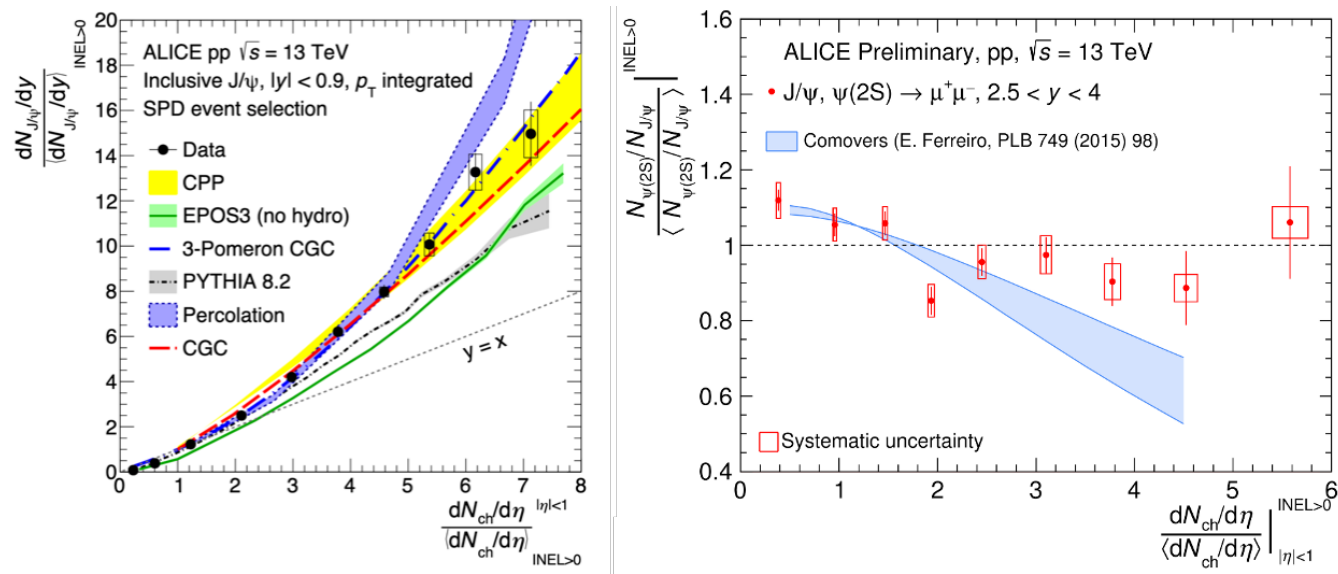

Figure 2: ALICE measurements of the multiplicity dependence of $J / \psi$ production [3] (left) and the $\psi(2 S) / J / \psi$ ratio (right).

The PHENIX collaboration has recently completed the analysis of $J / \psi$ production from the small system scan at RHIC, which included $p+\mathrm{Al}, p+\mathrm{Au}$, and ${ }^{3} \mathrm{He}+\mathrm{Au}$ data recorded at $\sqrt{s_{N N}}=$ $200 \mathrm{GeV}$ [8]. The $p_{T}$-integrated $J / \psi$ nuclear modification factor for each of these systems is shown as a function of rapidity in Fig. 3. Measurements in different rapidity bins probe different Bjorken $x$ values in the nucleus, and different time scales: in the $p$-going direction, the $c \bar{c}$ pair quickly exits the nucleus before hadronizing, however in the nucleus-going direction, the time spent inside the nucleus is comparable to the charmonium formation time.

At forward rapidity (in the $p$-going direction), in all systems, the data is consistent with calculations based on sampling from a modified parton distribution function (PDF) in the target nucleus. At backward rapidity, however, an additional suppression mechanism is required to match the data taken using the Au nucleus. When an absorption cross section is included to account for $J / \psi$ breakup while crossing the nucleus, the calculated suppression matches what is observed. 
It is interesting to note that similar measurements from LHCb and ALICE of $J / \psi$ production at backwards rapidity in $p+\mathrm{Pb}$ collisions are in good agreement with PDF calculations, without the need for any additional mechanisms $[9,10]$. The time the $c \bar{c}$ pair spends in the nucleus at the LHC is much smaller than at RHIC, due to the higher center-of-mass energy; therefore, absorption effects at the LHC are expected to be greatly reduced.

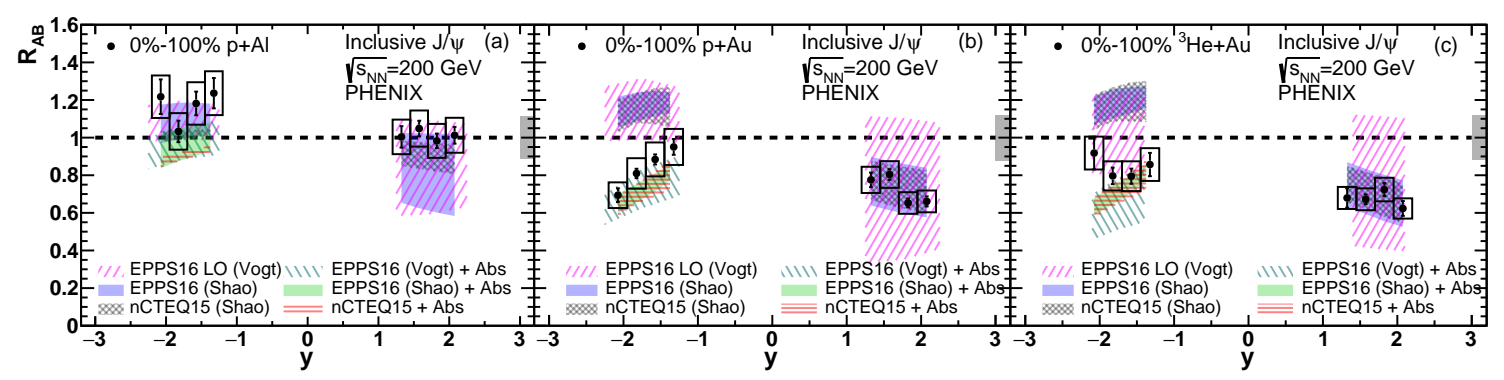

Figure 3: PHENIX measurements of the nuclear modification of $J / \psi$ mesons versus rapidity in $p+\mathrm{Al}$ (left), $p+\mathrm{Au}$ (center), and ${ }^{3} \mathrm{He}+\mathrm{Au}$ (right) collisions at $\sqrt{s_{N N}}=200 \mathrm{GeV}[8]$.


Figure 4: The measured $v_{2}$ of $J / \psi$ mesons in high-multiplicity $p+\mathrm{Pb}$ collisions [11] (left) and $\mathrm{Pb}+\mathrm{Pb}$ collisions [12] (right) at the LHC.

Another important metric for studying quarkonia is the elliptic flow amplitude $v_{2}$. Recent observations of significant flow in small systems have called the origin of flow effects into question. Measurements of $J / \psi v_{2}$ in high-multiplicity $p+\mathrm{Pb}$ collisions [11] have shown effects that are comparable to the $v_{2}$ observed in $\mathrm{Pb}+\mathrm{Pb}$ collisions [12], see Fig. 4. Transport model calculations that successfully describe the low $p_{T} v_{2}$ in $\mathrm{Pb}+\mathrm{Pb}$ collisions have been applied to $p+\mathrm{Pb}$ collisions, and do not give a $v_{2}$ amplitude that is sufficient to match the data, implying that mechanisms beyond final state collectivity may be at play [13]. A different calculation based on the CGC framework gives a significant $v_{2}$ due to correlations between gluons inside the target nucleus [14], although we note that similar CGC calculations have had mixed results describing light flavor $v_{2}$ in small systems.

Recently, the first measurements on $\Upsilon(1 S)$ flow in $\mathrm{Pb}+\mathrm{Pb}$ collisions at the LHC have been performed as shown in Fig. $5[15,16]$. While these first measurements have significant statis- 
tical uncertainties, they are consistent with zero. Transport models that successfully describe $\Upsilon$ suppression also give a $v_{2}$ that is near zero, due to the negligible production rate of $\Upsilon$ through recombination. One way to help resolve the major open question on the origin of collective flow is through measurements of $\Upsilon v_{2}$ in $p+\mathrm{Pb}$ collisions at the LHC. The previously described CGC calculations predict a substantial $v_{2}$ for $\Upsilon$ that is similar to what is observed for the $J / \psi$, while transport models are expected to give a very small $v_{2}$. Future data sets and analyses can provide decisive discrimination between these models.
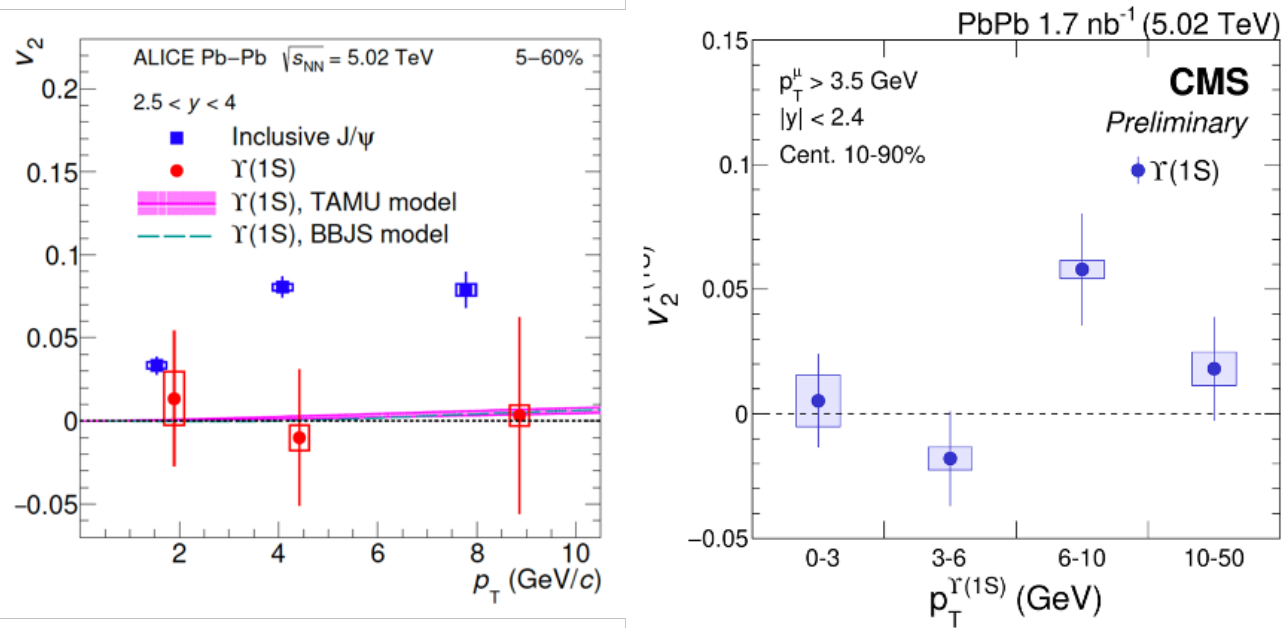

Figure 5: The elliptic flow amplitude $v_{2}$ of $\Upsilon(1 S)$ mesons in $\mathrm{Pb}+\mathrm{Pb}$ collisions at the LHC, from ALICE [15] (left) and CMS [16] (right)

\section{Exotic Hadrons in Medium}

The remarkable consistency between measurements of quarkonium and predictions from potential models was shaken in 2003, when the Belle experiment discovered the unexpected "charmoniumlike" X(3872) resonance in the invariant mass spectrum of $J / \psi \pi^{+} \pi^{-}$originating in the decays of $B$ hadrons [17]. The fact that the resonance was found as an intermediate state in the products of $B$ decays, and that the resonance itself had a $J / \psi$ in the final state, showed that it contained a $c \bar{c}$ pair. This plus the decay mode and narrow width of this resonance suggested a new charmonium state had perhaps been discovered $[18,19]$. However, the potential models which successfully predict the $Q \bar{Q}$ spectrum show that no charmonium states near the mass of $3872 \mathrm{MeV}$ with compatible quantum numbers $J^{P C}=1^{++}$are expected to exist [20]. The inability to reconcile the observed properties of the $X(3872)$ resonance with any expected $c \bar{c}$ states led to speculation that a multiquark state, consisting of at least two light quarks and a $c \bar{c}$ pair, had been found. Such "exotic" states were predicted by Gell-Mann and Zweig in the first papers proposing the quark model [21, 22], but unambiguous observations had proven elusive. Despite decades of measurements and theoretical interpretations, the exact nature of the $\mathrm{X}(3872)$ is not clear.

Embedding the $\mathrm{X}(3872)$ in the QCD medium provides new methods for probing its structure, as well as a new probe of the medium itself. Similar to conventional quarkonia states, the $\mathrm{X}(3872)$ 
can be disrupted via interactions with other produced particles, with a probability that is related to its binding energy. LHCb has studied the production of $\mathrm{X}(3872)$ relative to the well-known conventional charmonium state $\psi(2 S)$ as a function of multiplicity in $p+p$ collisions at $8 \mathrm{TeV}$ [23], as shown in the left panel of Fig. 6. A decrease of promptly produced X(3872) is observed relative to prompt $\psi(2 S)$, which may indicate that the $\mathrm{X}(3872)$ has a significantly weaker binding energy than the $\psi(2 S)$. Comparisons of this data with calculations from the Comover Interaction Model favor the compact tetraquark interpretation [24].

The CMS collaboration has measured the same ratio of $\mathrm{X}(3872) / \psi(2 S)$ cross sections $\mathrm{Pb}+\mathrm{Pb}$ collisions [25], which shows an increase relative to $p+p$ collisions, although with large uncertainties, in the right panel of Fig. 6. In this dense collision system, formation of tetraquarks via quark coalescence at chemical freezeout, or coalescence of $D^{0} \bar{D}^{0^{*}}$ into a molecular state at kinetic freezeout, may become an important production mechanism for the X(3872). Calculations based on coalescence [26] and AMPT transport [27] predict that a molecular X(3872) will be enhanced more than a compact tetraquark, while a different transport calculation comes to the opposite conclusion [28]. While current data does not give a clear discrimination between models, it is clear that this exotic state with an unprecedented number of constituent quarks poses a challenge to wellestablished theoretical models of heavy ion collisions. Additional data with reduced uncertainties across the full range of measureable multiplicities is required to definitively constrain models and draw firm conclusions.
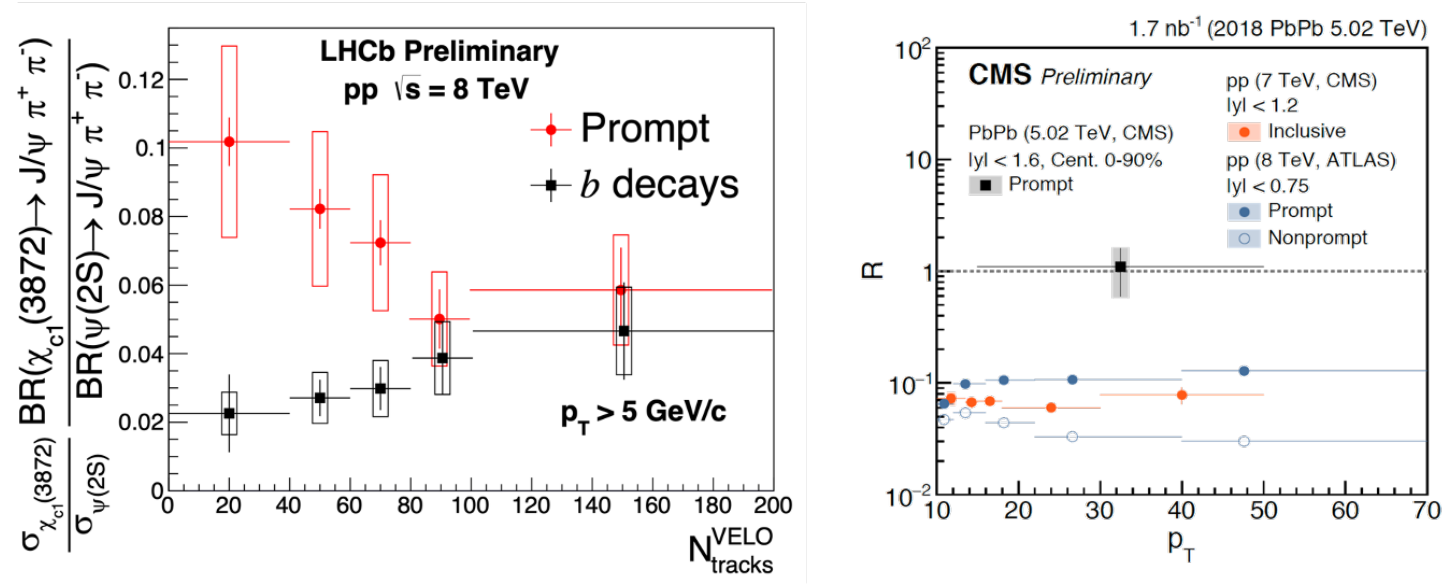

Figure 6: Preliminary results on modification of $\mathrm{X}(3872)$ production relative to $\psi(2 S)$ in $p+p$ collisions at $\mathrm{LHCb}$ (left) [23] and in $\mathrm{Pb}+\mathrm{Pb}$ collisions at CMS (right) [25].

\section{Future Facilities}

The LHCb collaboration is in the midst of upgrading their gaseous fixed-target System for the Measurement of Overlap with Gas (SMOG). A gas storage cell is being inserted in front of the LHCb VELO detector, which allows a greatly increased areal density of gas to be sampled by the beam. [29]. Rates of heavy quark production with the upgraded SMOG II system are significant, and measurements of exotic states such as $\mathrm{X}(3872)$ will also be pursued once the LHC resumes operations. 
Construction of the sPHENIX experiment is currently underway at Brookhaven. A major focus of the sPHENIX physics program is the measurement of the nuclear modification of the $\Upsilon(1 S)$, $2 S$, and $3 S$ states. Since the plasma temperatures achieved at RHIC is likely different from the temperatures at the LHC, this data will provide new constraints on models of bottomonia screening and transport in the QGP [30].

In the further future, the Electron Ion Collider (EIC) will allow the partonic structure of nuclei to be probed with unprecedented precision [31]. In the center-of-mass energy range where the EIC will operate, hadronization inside the nucleus is expected to become an important effect on observed particles [32]. Quarkonia produced at the EIC will therefore propagate through the medium of the cold nucleus as a fully formed state, in contrast to their production at RHIC and the LHC, where hadronization mostly takes place outside the nucleus. The low backgrounds produced in $e+$ A collisions will also allow measurements of states that are difficult to access in heavy ion collisions to be made at the EIC. Exposure to the nuclear environment offers new ways to probe exotic heavy quark states: for example, large, weakly bound molecular states should be disrupted more than tightly bound tetra- or pentaquark states as they cross the nucleus (see Fig. 7). Therefore, measurements of the nuclear modification of these states can discriminate between hadron structure models.
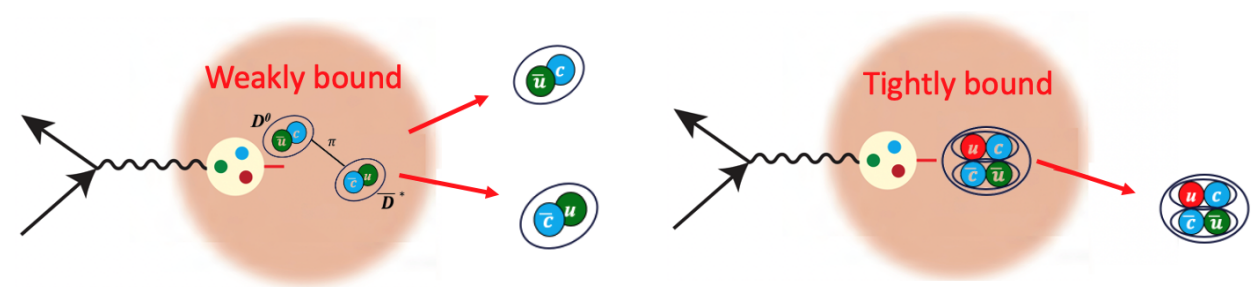

Figure 7: Measurements of exotic hadrons produced inside the nucleus at the EIC can discriminate between models of weakly bound states, such as hadronic molecules (left) and more tightly bound states, such as compact tetraquarks (right).

\section{Summary}

More than three decades after the seminal work of Matsui and Satz, quarkonia remains a central focus of heavy ion physics. Measurements of heavy quark states provide information on the initial state of the beam projectiles, interactions within and outside the nucleus, the dynamics of the plasma, and the allowed configurations of quarks bound inside hadrons. With improved data sets, upgrades, and future facilities, we expect greatly improved experimental constraints on many important questions in the near future. 


\section{References}

[1] T. Barnes, S. Godfrey, and E. S. Swanson. Higher charmonia. Phys. Rev. D, 72:054026, Sep 2005.

[2] Roel Aaij et al. Near-threshold $D \bar{D}$ spectroscopy and observation of a new charmonium state. JHEP, 07:035, 2019.

[3] Shreyasi Acharya et al. Multiplicity dependence of $\mathrm{J} / \psi$ production at midrapidity in pp collisions at $\sqrt{s}=13$ TeV. Phys. Lett., B:135758, 2020.

[4] B.Z. Kopeliovich, H.J. Pirner, I.K. Potashnikova, K. Reygers, and Iván Schmidt. J/ $\psi$ in high-multiplicity pp collisions: Lessons from pA collisions. Phys. Rev. D, 88(11):116002, 2013.

[5] Yan-Qing Ma, Prithwish Tribedy, Raju Venugopalan, and Kazuhiro Watanabe. Event engineering studies for heavy flavor production and hadronization in high multiplicity hadron-hadron and hadron-nucleus collisions. Phys. Rev. D, 98(7):074025, 2018.

[6] Eugene Levin, Iván Schmidt, and Marat Siddikov. Multiplicity dependence of quarkonia production in the CGC approach. Eur. Phys. J. C, 80(6):560, 2020.

[7] E.G. Ferreiro. Excited charmonium suppression in proton-nucleus collisions as a consequence of comovers. Phys. Lett., B749:98-103, 2015.

[8] U. Acharya et al. Measurement of $J / \psi$ at forward and backward rapidity in $p+p, p+A$, $p+A \mathrm{u}$, and ${ }^{3} \mathrm{He}+\mathrm{Au}$ collisions at $\sqrt{s_{N N}}=200 \mathrm{GeV}$. Phys. Rev. C, 102(1):014902, 2020.

[9] R. Aaij et al. Prompt and nonprompt $\mathrm{J} / \psi$ production and nuclear modification in $p \mathrm{~Pb}$ collisions at $\sqrt{s_{\mathrm{NN}}}=8.16 \mathrm{TeV}$. Phys. Lett. B, 774:159-178, 2017.

[10] Shreyasi Acharya et al. Inclusive $\mathrm{J} / \psi$ production at forward and backward rapidity in $\mathrm{p}-\mathrm{Pb}$ collisions at $\sqrt{s_{\mathrm{NN}}}=8.16 \mathrm{TeV}$. JHEP, 07:160, 2018 .

[11] Albert M Sirunyan et al. Observation of prompt $\mathrm{J} / \psi$ meson elliptic flow in high-multiplicity pPb collisions at $\sqrt{s_{\mathrm{NN}}}=8.16 \mathrm{TeV}$. Phys. Lett. B, 791:172-194, 2019.

[12] Shreyasi Acharya et al. $\mathrm{J} / \psi$ elliptic and triangular flow in $\mathrm{Pb}-\mathrm{Pb}$ collisions at $\sqrt{s_{\mathrm{NN}}}=5.02$ TeV. arXiv:2005.14518.

[13] Xiaojian Du and Ralf Rapp. In-Medium Charmonium Production in Proton-Nucleus Collisions. JHEP, 03:015, 2019.

[14] Cheng Zhang, Cyrille Marquet, Guang-You Qin, Shu-Yi Wei, and Bo-Wen Xiao. Elliptic Flow of Heavy Quarkonia in pA Collisions. Phys. Rev. Lett., 122(17):172302, 2019.

[15] Shreyasi Acharya et al. Measurement of $\Upsilon(1 \mathrm{~S})$ elliptic flow at forward rapidity in $\mathrm{Pb}-\mathrm{Pb}$ collisions at $\sqrt{s_{\mathrm{NN}}}=5.02 \mathrm{TeV}$. Phys. Rev. Lett., 123(19):192301, 2019. 
[16] CMS Collaboration. Measurement of the elliptic flow of $\Upsilon(1 \mathrm{~S})$ and $\Upsilon(2 \mathrm{~S})$ mesons in $\mathrm{PbPb}$ collisions at $\sqrt{\mathrm{s}_{\mathrm{NN}}}=5.02 \mathrm{TeV}$. CMS-PAS-HIN-19-002, 2019.

[17] S.-K. Choi et al. Observation of a narrow charmoniumlike state in exclusive $B^{ \pm} \rightarrow$ $K^{ \pm} \pi^{+} \pi^{-} J / \psi$ decays. Phys. Rev. Lett., 91:262001, Dec 2003.

[18] Ted Barnes and Stephen Godfrey. Charmonium options for the X(3872). Phys. Rev. D, 69:054008, Mar 2004.

[19] Estia J. Eichten, Kenneth Lane, and Chris Quigg. Charmonium levels near threshold and the narrow state $X(3872) \rightarrow \pi^{+} \pi^{-} J / \psi$. Phys. Rev. D, 69:094019, May 2004.

[20] R. Aaij et al. Determination of the $X(3872)$ meson quantum numbers. Phys. Rev. Lett., 110:222001, 2013.

[21] M. Gell-Mann. A schematic model of baryons and mesons. Physics Letters, 8(3):214 - 215, 1964.

[22] G. Zweig, CERN Report No. CERN-TH-401. An SU(3) model for strong interaction symmetry and its breaking. 1964.

[23] LHCb Collaboration. Multiplicity-dependent modification of $\chi_{c 1}$ (3872) and $\psi(2 \mathrm{~S})$ production in $p p$ collisions at $\sqrt{s}=8 \mathrm{TeV}$. LHCB-CONF-2019-005, 2019 .

[24] Angelo Esposito, Elena G. Ferreiro, Alessandro Pilloni, Antonio D. Polosa, and Carlos A. Salgado. The nature of $X(3872)$ from high-multiplicity $p p$ collisions. arXiv:2006.15044.

[25] CMS Collaboration. Evidence for $\chi_{c 1}(3872)$ in $\mathrm{PbPb}$ collisions and studies of its prompt production at $\sqrt{s_{\mathrm{NN}}}=5.02 \mathrm{TeV}$, CMS-PAS-HIN-19-005. 2019.

[26] Sungtae Cho et al. Multi-quark hadrons from Heavy Ion Collisions. Phys. Rev. Lett., 106:212001, 2011.

[27] Hui Zhang, Jinfeng Liao, Enke Wang, Qian Wang, and Hongxi Xing. Deciphering the nature of X(3872) in heavy ion collisions. arXiv:2004.00024.

[28] Biaogang Wu, Xiaojian Du, Matthew Sibila, and Ralf Rapp. X(3872) Transport in Heavy-Ion Collisions. arXiv:2006.09945.

[29] LHCb Collaboration. LHCb SMOG Upgrade. Technical Report CERN-LHCC-2019-005. LHCB-TDR-020, CERN, Geneva, May 2019.

[30] A. Adare et al. An Upgrade Proposal from the PHENIX Collaboration. arXiv:1501.06197.

[31] A. Accardi et al. Electron Ion Collider: The Next QCD Frontier: Understanding the glue that binds us all. Eur. Phys. J. A, 52(9):268, 2016.

[32] Ivan Vitev. Radiative processes and jet modification at the EIC. In Probing Nucleons and Nuclei in High Energy Collisions: Dedicated to the Physics of the Electron Ion Collider, pages 244-247, 2020. 